of models of these systems to membrane receptors. In contrast, S. Ebashi (Tokyo), lecturing on calcium ions as a basis of pharmacological action, related his account to functional and pharmacological problems, such as the mechanism of caffeine contractions in striated muscle, and the role of calcium ions in excitable membranes and in triggering metabolic pathways.

R. Y. Ilyutchenok (Novosibirsk) lectured on the action of drugs on memory and learning. The assumptions involved in any analytical (rather than simply descriptive) work in this area seem to be rather great. For example, if a drug had an effect on learning, and if it bchaved as an acetylcholine antagonist at the periphery, the interpretation was that a cholinergic pathway was involved (directly or indirectly) in learning; but this involves the assumption that the antagonist is working in the same way in the central nervous system as at the periphery. Many such drugs, however, have different effects on different tissues: indeed, different receptors to the same transmitter substance (such as adrenergic $\alpha$ and $\beta$-receptors) are categorized chiefly on just such differences. Clearly, negative results in such experiments would not rule out the involvement of a cholinergic pathway, making positive results hard to assess.

An entirely different approach to central nervous system function was described by D. R. Curtis (Canberra). His aim was to test the hypothesis that certain. pharmacologically active amino-acids present in various parts of the central nervous system function as neurotransmitters. Responses to ionophoretically applied amino-acids were compared with synaptically evoked excitatory post synaptic potentials (EPSPs) and inhibitory post synaptic potentials (IPSPs), in various conditions designed to distinguish between exogenously applied amino-acid and endogenous transmitter, if these were not identical substances. Antagonists proved to be particularly useful tools in this respect: for example, the IPSP of some cells (particularly in the ventral horns of the spinal cord, where glycine is present in large amounts) is abolished by strychnine. Both $\gamma$-amino butyric acid (GABA) and glycine hyperpolarize these cells in normal conditions, but the response to glycine is abolished by strychnine while that to GABA is not, thus excluding the possibility that GABA is the inhibitory transmitter while leaving the case for glycine intact.

In conclusion, insights were offered into many exciting areas of pharmacology and related ficlds, and the failure of the discussion gatherings was largely compensated for by the several excellent symposia, trigger mectings and demonstrations.

\section{CHEMICAL STRUCTURE}

\section{Liquid Crystals}

\section{from a Correspondent}

When a conference on liquid crystals was held at Woolwich Polytechnic on June 12 and 13, G. W. Gray (University of Hull) showed that changes in the thermal stabilities of liquid crystals can often be rationalized in simple structural terms. He was outlining features of molecular geometry and structure which determine whether a compound will be liquid crystalline. $\mathrm{He}$ emphasized that the racemic analogue of an optically active cholesteric compound is nematic in behaviour and went on to point out some uses and applications of liquid crystals.

Conmar Robinson (who is still active although formally retired) considered the remarkable packing arrangement in smectic liquid crystals, as revealed by their focal conic texture. Cholesteric liquid crystals formed in solutions of certain polypeptides show visible periodicities equal to half the pitch of the axis of torsion. Where, in other cases, the pitch is comparable with the wavelength of light, the solutions exhibit "cholesteric" colours and the reflected light is circularly polarized. It is interesting that the light reflected from certain iridescent beetles is also circularly polarized. The cuticles of these and of other arthropods contain similar" "cholesteric" structures.

A. C. Neville (ARC Unit of Insect Physiology, Oxford) reported ultrastructural evidence which supports the hypothesis of helicoidal architecture of cholesteric liquid crystals for insect skeletal materials. The solid state helicoidal cuticle probably arises by crystallization of a cholesteric liquid crystal system of chitin and protein.

Professor A. S. C. Lawrence (University of Sheffield) discussed lyotropic mesomorphs, which consist of a solvent, usually water, and a molecule with structurebuilding properties. Most work has been done on those consisting of one or more hydrophobic chains plus one or more hydrophilic polar groups, ionic or non-ionic, which interact locally with water and layer structure builders. They include soaps and the biologically important phosphatides. The temperature range of mesomorphic behaviour is limited by the upper transition to liquid and a lower transition at which the substance crystallizes in its low temperature low symmetry form with ejection of the water. The fatty chain is capable of numerous modes of internal thermal motion in the solid state which cause change of molecular shape leading to discontinuous changes of packing, and so to polymorphism. 'These transitions and the effect of water on them are studied by slow cooling curves, differential thermal analysis and polarized light microscopy with heating or cooling stage; identification is by infrared, NMR and X-ray diffraction.

J. S. Clunie (Procter and Gamble Ltd) outlined microscopy and diffraction results which make it possible to postulate plausible structures for various mesomorphic phases. He showed some visual confirmations achieved by electron microscopy. The use of X-ray techniques has been stimulated by the toroidal camera devised by $\Lambda$. Elliot (King's College, London), who described how it is used to study liquid crystals in poly- $\gamma$-benzyl glutamate solutions.

D. J. Morantz (Woolwich Polytechnic) proposed that the general approach to liquid crystals can be considered in the context of the structure of "crystalline liquids". This implies a three dimensional bulk structure. Phosphorescence and conductivity studies were reported which suggest stoichiometric physical complexes in certain alcohol/hydrocarbon liquid mixtures.

The detailed study of liquid crystal structures by electron spin resonance techniques was outlined by G. R. Luckhurst (University of Southampton). In a magnetic field the long axes of molecules of a nematic mesophase tend to be aligned parallel to the fiold. 
The degree of alignment or order in the mesophase decreases with increasing temperature until at the nematic-isotropic transition point it vanishes. These changes can be explained theoretically by deriving the pseudo-potential resulting from all the interactions between one molecule and the remainder. Electron resonance has shown that a model for the potential based on dispersion forces is inadequate. Furthermore, direct determination of the form of the pseudo-potential suggests that repulsive forces may dominate.

Professor E. J. Ambrose (Chester Beatty Research Institute) discussed the biological importance of mesomorphic states in terms of the function of the cell membrane and the cell surface complex. Several examples were shown of cell membrane changes in animated and still form. Discussion gave rise to the suggestion that carcinogens can interfere with the liquid crystal structure of the membrane.

\section{SONIC BOOMS}

\section{Seismic Effects}

\section{from our Geomagnetism Correspondent}

THE debate on the merits and demerits of civil supersonic flight takes place at several levels - sociological, psychological, financial and even philosophical-but all too frequently in the absence of reliable scientific data. McDonald and Goforth (J. Geophys. Res., 74, 2637 ; 1969) have attempted to remedy this deficiency as far as sonic booms are concerned by simply observing ground particle velocities produced during the supersonic flight of existing military aircraft. The basic characteristics of the four military aircraft monitored are shown in the table together with similar details of Concorde and the proposed Boeing SST for comparison.

$$
\begin{aligned}
& \text { CHARACTERISTICS OF FOUR MILITARY AIRCRAFT, COM- } \\
& \text { PARED WITII CONCORDE AND BOEING SST } \\
& \text { Aircraft }
\end{aligned}
$$

Particle velocities were measured on portable seismograph stations set up at Edwards Air Force Base, California (EAFB), the Tonto Forest Seismological Observatory near Payson, Arizona (TFSO) and the Uinta Basin Seismological Observatory near Vernal, Utah (UBSO). In addition, modified observatory equipment was used at TFSO and UBSO. In all, 183 missions were monitored at EAFB. Eight missions at TFSO and three at UBSO allowed a comparison of sonic boom seismic effects over different geological units; and a deep vault at UBSO made possible a study of signal attenuation with depth.

What emerged from an analysis of the field data was that both the magnitude and areal extent of the seismic effects were extromely limited. Horizontal displace- ments were largely confined to the boom pressure envelope, and vertical displacements to the upper few metres of the Earth's crust. Maximum ground particle velocities were associated with the leading and trailing edges of the acoustic $\mathrm{N}$ waves; and the overall maximum particle velocity observed in the whole series of experiments was $600 \mathrm{microns} / \mathrm{second}$. Up to overpressures of $25 \mathrm{~kg} / \mathrm{m}^{2}$, the maximum particle velocity varied linearly with maximum overpressure but was independent of aircraft altitude, speed and type, temperature, wind velocity and relative humidity except in so far as these factors influence the overpressure itself.

McDonald and Goforth find it instructive to compare the observed maximum particle velocity of 600 microns/second with publicly recommended maximum levels of vibration. The US Bureau of Mines, for example, recommends a maximum peak particle velocity of 50,800 microns/second. Similarly, the US Corps of Engineers, the New York State Power Authority and the States of Massachusctts and New Jersey only consider particle velocities of less than 25,400 microns/second to be safe. In short, the maximum particle velocity observed by McDonald and Goforth on relatively loose materials was less than three per cent of currently acceptable building damage standards.

The nominal overpressures (but not the overpressures from "superbooms" produced by the simultaneous arrival of two or more booms from different points in the flight path) expected from Concorde and the Boeing SST are less than $25 \mathrm{~kg} / \mathrm{m}^{2}$, that is, within the range of overpressures monitored by McDonald and Goforth. The anti-SST lobby is thus not likely to be very happy about these results, but will no doubt point to the wealth of evidence for physical damage already produced by military supersonic overflying in the USA. Presumably the explanation for the conflict here is that damage is limited to particularly weak structures such as historic buildings and particularly vulnerable sub-structures such as windows.

\section{MATERIALS \\ Abrasive Wear}

\section{from a Correspondent}

Delegates to the Materials Science Club conference at the National College of Agricultural Engineering, Silsoe, on July 16 and 17 found that there is an important link between the life of both motor car tyres and agricultural ploughs, the design of toothpaste and such down to earth matters as shoe leather. The link is, of course, abrasive wear, a somewhat neglected topic that is currently recognized to have fundamental significance in materials science and metallurgy, and which provided the theme of the conference.

The term abrasive wear was not interpreted narrowly, for some of the different modes of wear that may qualify as abrasive still require exploration. First, the wear process was discussed as far as possible in physical terms. R. C. D. Richardson (National Institute of Agricultural Engineering) defined abrasion as the removal of material by hard particles on a minute scale by a cutting process. The angle of attack is of great importance in that it either causes a groove on the surface or the surface is chipped, as in true abrasion. 